\title{
Sífilis materna e congênita: um retrato do cenário brasileiro
}

\author{
Maternal and congenital syphilis: a portrait of the brazilian scenery \\ Sífilis materna y congénita: un retrato del escenario brasileño
}

\begin{abstract}
RESUMO
Objetivo: analisar a incidência de sífilis em gestantes e congênitas, notificadas no Brasil de 2008 a 2017. Método: Os dados referentes a número e características dos casos, foram coletados no Sistema de Informação de Agravos de Notificação, para fins de cálculo das taxas. Os dados extraídos foram tabulados e posteriormente analisados por meio de estatística descritiva. Resultados: Em 2017, 24.668 casos de sífilis congênita foram notificados, demonstrando aumento de 16,4\% nas notificações em relação ao ano anterior, com uma taxa de incidência de 8,2 casos por 1000 nascidos vivos. Evidenciando a fragilidade no acompanhamento pré-natal materno e do parceiro sexual, especialmente para as populações mais vulneráveis com condições econômicas desfavoráveis, baixa escolaridade e dificuldades no acesso à saúde. Conclusão: Os atuais esforços governamentais são insuficientes para controlar o agravo e as iniquidades sociais, denotando a necessidade de revisão estratégias em prol da qualidade e eficiência do acompanhamento pré-natal.
\end{abstract}

DESCRITORES: Sífilis; Sífilis congênita; Transmissão vertical de doenças infecciosas; Saúde materno-infantil

\section{ABSTRACT}

Objective: to analyze the incidence of syphilis in pregnant and congenital women notified in Brazil from 2008 to 2017. Method: Data regarding the number and characteristics of cases were collected in the Notifiable Diseases Information System, for the purposes of calculating rates. The extracted data were tabulated and later analyzed using descriptive statistics. Results: In 2017, 24,668 cases of congenital syphilis were notified, showing an increase of $16.4 \%$ in notifications compared to the previous year, with an incidence rate of 8.2 cases per 1000 live births. Evidencing the fragility of prenatal care for the mother and the sexual partner, especially for the most vulnerable populations with unfavorable economic conditions, low education levels and difficulties in accessing health care. Conclusion: Current government efforts are insufficient to control the problem and social inequities, denoting the need to review strategies in favor of the quality and efficiency of prenatal care

DESCRIPTORS: Syphilis; Congenital syphilis; Vertical infectious disease transmission; Maternal and child health

\section{RESUMEN}

Objetivo: analizar la incidencia de sífilis en gestantes y congénitas notificadas en Brasil de 2008 a 2017. Método: Los datos sobre el número y características de los casos fueron recolectados en el Sistema de Información de Enfermedades Notificables, con el propósito de calcular las tasas. Los datos extraídos fueron tabulados y posteriormente analizados mediante estadística descriptiva. Resultados: En 2017 se notificaron 24.668 casos de sífilis congénita, mostrando un aumento del 16,4\% en las notificaciones respecto al año anterior, con una tasa de incidencia de 8,2 casos por 1000 nacidos vivos. Evidenciando la fragilidad de la atención prenatal para la madre y la pareja sexual, especialmente para las poblaciones más vulnerables con condiciones económicas desfavorables, bajos niveles de educación y dificultades para acceder a la atención de salud. Conclusión: Los esfuerzos gubernamentales actuales son insuficientes para controlar el problema y las inequidades sociales, lo que denota la necesidad de revisar estrategias a favor de la calidad y eficiencia de la atención prenatal.

DESCRIPTORES: Sífilis; Sífilis congénita; Transmisión vertical de enfermedad infecciosa; Salud materno-infantil.

RECEBIDO EM: 12/10/2021 APROVADO EM: 18/11/2021

\section{Neide Mayumi Mitsugui}

Farmacêutica especialista em Gestão em Saúde na Secretaria Municipal de Saúde de Maringá. Maringá, Paraná, Brasil.

ORCID: 0000-0001-7074-9963 


\section{Bianca Machado Cruz Shibukawa}

Enfermeira. Mestra em Enfermagem. Professora do Departamento de Enfermagem da Universidade Estadual de Maringá/ UEM, Maringá, Paraná, Brasil

ORCID: 0000-0002-7739-7881

\section{Rosimara Oliveira Queiroz}

Enfermeira. Mestra em Enfermagem. Doutoranda do Programa de PósGraduação em Enfermagem da Universidade Estadual de Maringá/UEM, Maringá, Paraná, Brasil.

ORCID: 0000-0001-7976-2259

\section{Camila Moraes Garollo Piran}

Enfermeira. Mestranda do Programa de Pós-Graduação em Enfermagem da Universidade Estadual de Maringá/UEM, Maringá, Paraná, Brasil.

ORCID: 0000-0002-9111-9992

\section{Marcela Demitto Furtado}

Enfermeira. Doutora em Enfermagem. Professora no Programa de PósGraduação em Enfermagem da Universidade Estadual de Maringá. Maringá, Paraná, Brasil.

ORCID: 0000-0003-1427-4478

\section{leda Harumi Higarashi}

Enfermeira. Doutora em Enfermagem. Professora Programa de Pós-Graduação em Enfermagem da Universidade Estadual de Maringá. Maringá, Paraná, Brasil.

ORCID: 0000-0002-4205-6841

\section{INTRODUÇÃO}

A sífilis é uma infecção bacteriana, de caráter sistêmico, evolução crônica e muitas vezes assintomática, causada pela bactéria espiroqueta Treponema pallidum, que se apresenta nas formas adquirida e congênita. A sífilis adquirida pode ser contraída sexualmente ou transfusão sanguínea, já a sífilis congênita ocorre por transmissão vertical ${ }^{1}$.

A notificação compulsória da sífilis congênita concretizou-se no Brasil por meio da Portaria $n^{\circ} 542$ de 1986 , e, o compromisso internacional para eliminar a sífilis congênita, foi assumido através da Resolução CE 116/14 da Organização Pan-Americana da Saúde (OPAS) ${ }^{2}$.

Apesar do compromisso global com a erradicação da sífilis, a Organização Mundial de Saúde (OMS), declarou em 2017 que a sífilis atingiu 12 milhões de pessoas no mundo, permitindo estimar-se que, anualmente, 930.000 mulheres grávidas possam ter siffilis transmissível durante a gravidez, resultando em aproximadamente 350.000 resultados adversos no nascimento ${ }^{3}$.

No Brasil, em 2016, a sífilis foi declarada

\section{A sífilis adquirida pode ser contraída sexualmente ou transfusão} sanguínea, já a sífilis congênita ocorre por transmissão vertical. um grave problema de saúde pública, e os indicadores epidemiológicos apontavam para um cenário de epidemia deste agravo 4 . Conquanto esforços governamentais, em várias regiões do Brasil, identificaram-se dificuldades para controle da epidemia de sífilis como: problemas estruturais e de recursos humanos para a implementação de testes rápidos para sífilis ofertada na Atenção Básica, falhas na assistência pré-natal, necessidade de novas estratégias para reduzir a transmissão vertical da sífilis, além de dificuldades relacionadas ao tratamento de parceiros sexuais das gestantes com sífilis, imprescindível para evitar a reinfecção da gestante $^{5-6}$.

Sendo assim, torna-se importante a observação dos casos de sífilis adquirida ou congênita, com a finalidade de melhorar as estratégias de prevenção, oferta de testes rápidos, além da detecção e tratamento precoces.

Diante disso, as equipes de saúde da atenção primária têm um papel muito importante pela aproximação com o paciente, consequentemente, podem estar colaborando com a mudança do perfil epidemiológico da sífilis gestacional e congênita ${ }^{6}$. 


\section{artigo}

Frente ao exposto, considerando a prevenção da transmissão vertical da sífilis como condição prioritária de atenção no Ministério de Saúde, conforme Agenda de Ações Estratégicas para Redução da Sífilis Congênita no Brasil, o objetivo deste estudo é caracterizar o cenário nacional da sífilis gestacional e congênita no período de 2008 a 2017.

\section{MÉTODO}

Estudo transversal de caráter ecológico e abordagem quantitativa, que investigou os casos notificados de sífilis em gestantes e de sífilis congênita no país e por região, durante o período de 2008 a 2017.

A população do estudo foram todas as fichas de notificação de sífilis gestacional e congênita, registradas na plataforma online do Departamento de Vigilância, Prevenção e Controle das Infecções Sexualmente Transmissíveis, do HIV/Aids e Hepatites Virais, da Secretaria de Vigilância em Saúde, do Ministério de Saúde, de 2008 a 2017.

Para a coleta de dados, utilizou-se as informações disponibilizados pela Plataforma online do Departamento de Vigilância, Prevenção e Controle das Infecções Sexualmente Transmissíveis, do HIV/Aids e Hepatites Virais, da Secretaria de Vigilância em Saúde, do Ministério de Saúde (DIAHV/MS/SVS).

Realizou-se a coleta de dados nos meses de dezembro de 2018 a janeiro de 2019, por meio de questionário estruturado, selecionou-se as variáveis: número de casos, taxa de detecção da síflis em gestante e sífilis congênita, características sociodemográficas maternas (faixa etária, escolaridade e raça/cor) e características do acompanhamento pré-natal (realização de pré-natal, momento do diagnóstico da sífilis, esquema de tratamento da mãe e de seu parceiro).

Tabulou-se e analisou-se os dados por meio do Programa Microsoft Excel 2016 e R versão 3.6.3. Os dados analisados foram expressos em taxas, números absolutos e percentuais. Destes, a taxa de detecção de sífilis em gestantes foi calculada utilizando-se o número de notificações de sífilis em gestante sobre o número de nascidos vivos, obtidos no Sistema de Informação sobre Nascidos Vivos (SINASC), no mesmo ano; a taxa de incidência de sífilis congênita foi calculada como a razão entre o número de notificações e número de nascidos vivos por 1.000 nascidos vivos no mesmo ano.

Dispensou-se a apreciação ética pelo Comitê Permanente de Ética em Pesquisa Envolvendo Seres Humanos, com base nas Resoluções 466(7) e 510(8) do Conselho Nacional de Saúde do Ministério da Saúde, por se tratar de banco de dados de domínio público e irrestrito.

\section{RESULTADOS}

Durante o período de 2008 a 2017, houve aumento significativo no número de casos de notificações de sífilis gestacional, sendo que em 2008 houve 7.306 notificações, e em 2017, 49.028, representando aumento de $671 \%$ do número de notificações compulsórias durante os dez anos contemplados pelo estudo. A taxa de detecção da doença também apresentou aumento, em 2017, destacaram-se as regiões Sudeste $(19,6)$ e Sul $(19,3)$, com valores superiores à média nacional $(16,2)$ de taxa de detecção de gestantes com sífilis (Figura 1).

No ano de 2008 foram notificados 5.745 casos de sífilis congênita e, em 2017, foram notificados 24.668 casos de sífilis congênita no país, resultando num aumento de $429 \%$ de notificações nos dez anos do estudo. A taxa de detecção da doença também apresentou aumento, em contrapartida, as taxas de detecção de sífilis congênita nas regiões Nordeste $(8,1)$, Sudeste $(8,9)$ e Sul $(8,8)$ foram, aproximadamente, similares a média nacional $(8,2)$ (Figura 2).

Em relação às características sociodemográficas das mães das crianças notificadas com sífilis congênita e as mulheres encaminhadas por sífilis gestacional, $71,8 \%$ e $71,5 \%$ das possuíam idade entre 20 e 39 anos, respectivamente. Quanto ao critério escolaridade, $47 \%$ das mães das crianças com sífilis congênita e $56,3 \%$ das mulheres com sífilis gestacional, possuíam menos de oito anos de estudo. Em relação à raça/cor, em aproximadamente $66 \%$ de ambos os casos as mulheres não eram brancas. Ressalta-se ainda o grande número de informações ignoradas no momento do preenchimento da notificação compulsória, que alcançou a marca de $28,9 \%$ de falta de registro de informação no campo de escolaridade (Tabela 1 ).

Em relação ao acompanhamento pré-natal das mulheres que transmitiram a

\section{Figura 1. Taxa de detecção de sífilis gestacional no Brasil por regiões, no período}

de 2008 a 2017. Maringá-PR, 2019.

\begin{tabular}{|c|c|c|c|c|c|c|c|c|c|c|}
\hline 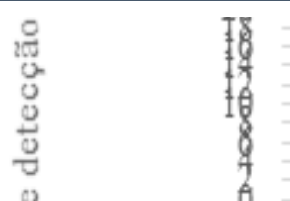 & $\therefore$ & $=$ & - & & $E$ & & & & & \\
\hline 0 & 200 & 200 & 201 & 201 & 201 & 201 & 201 & 201 & 201 & 201 \\
\hline & 8 & 9 & 0 & 1 & 2 & 3 & 4 & 5 & 6 & 7 \\
\hline -Brasil & 2,5 & 2,9 & 3,5 & 4,7 & 5,7 & 7,2 & 8,9 & 10,8 & 12,6 & 16,2 \\
\hline- Norte & 3,7 & 4,1 & 4,2 & 4,8 & 4,9 & 6,2 & 7,7 & 10,2 & 12,1 & 14,6 \\
\hline$\leftarrow$ Nordeste & 2 & 2,3 & 2,8 & 3,8 & 4,3 & 5,2 & 6,2 & 7 & 7,8 & 10,7 \\
\hline -Sudeste & 2,3 & 2,8 & 3,8 & 5,6 & 6,9 & 8,7 & 10,9 & 12,5 & 15,1 & 19,6 \\
\hline$\Rightarrow$ Sul & 1,8 & 2,3 & 2,7 & 3,8 & 5,1 & 7,1 & 9,7 & 14,7 & 16,6 & 19,3 \\
\hline- Centro-Oeste & 4,7 & 5 & 5,3 & 5,4 & 6,1 & 8,2 & 9,3 & 10,5 & 11,6 & 15,8 \\
\hline
\end{tabular}

Fonte: DIAHV/MS/SVS, 2019 
Figura 2. Taxa de detecção de sífilis congênita no Brasil por regiões, no período de 2008 a 2017. Maringá-PR, 2019.

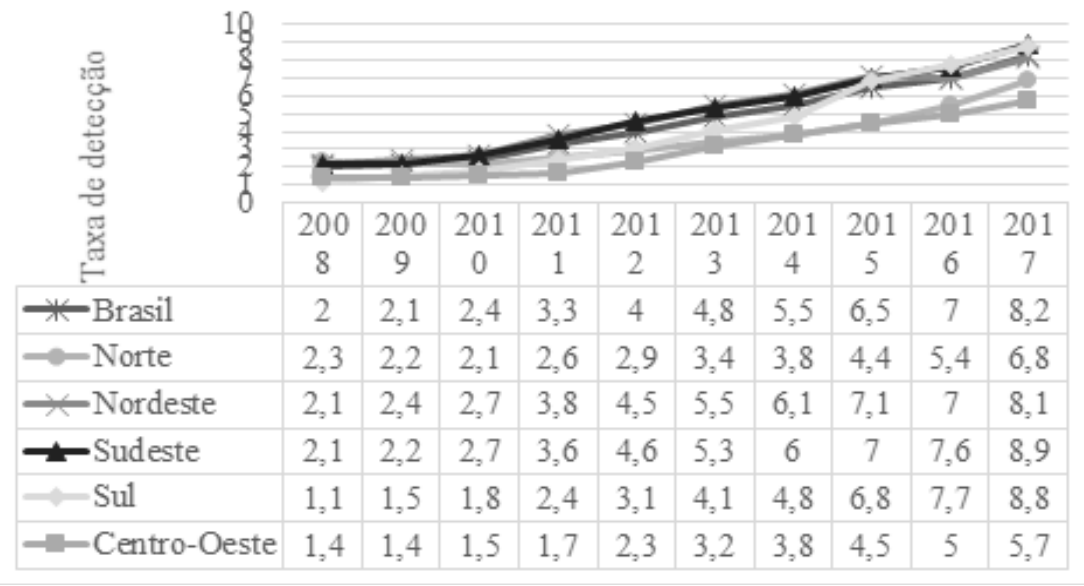

Fonte: DIAHV/MS/SVS, 2019.

Tabela 1. Perfil sociodemográfico das mães das crianças notificadas com sífilis congênita e as mulheres encaminhadas por sífilis gestacional, no período de 2008 a 2017 no Brasil.

\begin{tabular}{|l|l|l|l|l|}
\hline VARIÁVEIS MATERNAS & \multicolumn{2}{|c|}{ SÍFILIS CONGÊNITA } & \multicolumn{2}{l|}{ SÍFILIS GESTACIONAL } \\
\hline Faixa Etária & N & $\%$ & N & $\%$ \\
\hline$<15$ anos & 1796 & 1,0 & 1364 & 1,0 \\
\hline 15 a 19 anos & 39.562 & 22,5 & 31178 & 22,9 \\
\hline 20 a 39 anos & 126.483 & 71,8 & 97.301 & 71,5 \\
\hline$>40$ anos & 3810 & 2,2 & 2836 & 2,1 \\
\hline Ignorado & 4457 & 2,5 & 3421 & 2,5 \\
\hline Escolaridade & & & & \\
\hline Analfabeto & 2256 & 1,3 & 1505 & 1,1 \\
\hline$<8$ anos de estudo & 82755 & 47,0 & 76633 & 56,3 \\
\hline$>8$ anos de estudo & 40313 & 22,9 & 18679 & 13,7 \\
\hline Ignorado & 50784 & 28,8 & 39283 & 28,9 \\
\hline Raça ou Cor & & & & \\
\hline Branca & 41.352 & 23,5 & 32386 & 23,8 \\
\hline Não branca & 116.272 & 66,0 & 89932 & 66,1 \\
\hline Ignorada & 18484 & 10,5 & 13782 & 10,1 \\
\hline Fonte: DIAHV/MS/SVS, 2019. & \multicolumn{5}{|l}{} \\
\hline
\end{tabular}

doença para seus filhos, verificou-se que no segundo quinquênio, houve aumento na realização do pré-natal em $6 \%$. O momento de detecção da sífilis aumentou no momento do pré-natal em $11,6 \%$ e diminuiu $8,1 \%$ no momento do parto e/ou curetagem.

$\mathrm{O}$ tratamento inadequado ainda é predominante e crescente, visto que houve
Os resultados demonstraram que houve um progressivo aumento nas taxas de detecção de sífilis em gestante e sífilis congênita no Brasil, durante o período 2008 a 2017. Segundo o Boletim Epidemiológico de Sífilis 2018, do Ministério da Saúde, este aumento pode ser atribuído, em parte, ao aprimoramento do sistema de notificação, no SINAN, e à ampliação de oferta de teste rápido de sífilis na Atenção Básica?.

Este fato foi constatado em estudo descritivo dos casos notificados de sífilis em gestantes e congênita, a partir de dados do SINAN, de 2007 a 2012, no qual os autores concluíram que incrementos das taxas de detecção de sífilis nas unidades federativas Amazonas, Ceará, Espírito Santo, Rio de Janeiro, Rio Grande do Sul e Distrito Federal, localidades com representatividade regional e condições diversas de acesso à saúde, podem ter sido impulsionados pelo aumento de notificações de casos no SINAN. Além disso, nesse estudo, em todos os estados estudados, foi observado um aumento de utilização do teste rápido. Apenas no Distrito Federal foi verificada uma redução ${ }^{10}$.

Não obstante as evidências de aumento nas taxas de detecção da sífilis em gestante e congênita, há que se observar que a subnotificação ainda é uma realidade, como foi constatado em Minas Gerais. O referido estudo se iniciou com identificação de fichas de notificação epidemiológica, seguida de busca ativa nas maternidades, no período de 2007 a $2013^{11}$. A falta de notificação é um grande desafio para a vigilância epidemiológica da sífilis, não permitindo investigação de casos suspeitos, inviabilizando, assim, o adequado tratamento materno-infantil ${ }^{12}$.

Em relação à sífilis em gestante, em 2017, no Brasil, o total de casos notificados de sífilis em gestantes foi de 49.028, o que representou aproximadamente $28,5 \%$ mais casos que no ano anterior e a taxa de detecção foi 16,2 casos de sífilis em gestantes a cada 1.000 nascidos vivos. Convém ressaltar que a partir de 2017, o aumento no número de casos de sífilis em gestantes possivelmente esteja relacionado, ainda que em parte, às alterações quanto aos critérios 
Tabela 2. Acompanhamento pré-natal e tratamento da mulher e seu parceiro, no período de 2008 a 2017 no Brasil.

\begin{tabular}{|l|l|l|l|l|}
\hline \multirow{2}{*}{ REALIZAÇÃo PRÉ-NATAL } & \multicolumn{3}{|c|}{$2008-2012$} & \multicolumn{2}{c|}{$2013-2017$} \\
\cline { 2 - 5 } & $\mathrm{N}$ & $\%$ & $\mathrm{~N}$ & $\%$ \\
\hline Sim & 29.268 & 73,2 & 76.130 & 79,2 \\
\hline Não & 7.923 & 19,8 & 14.380 & 15,0 \\
\hline Ignorado & 2.817 & 7,0 & 5.582 & 5,8 \\
\hline $\begin{array}{l}\text { Momento do diagnóstico da } \\
\text { sífilis materna }\end{array}$ & & & & \\
\hline Durante o pré-natal & 16.543 & 41,3 & 50.860 & 52,9 \\
\hline No parto/curetagem & 16.794 & 42,0 & 32.540 & 33,9 \\
\hline Após o parto & 4.571 & 11,4 & 7.936 & 8,3 \\
\hline Não realizado & 256 & 0,6 & 586 & 0,6 \\
\hline Ignorado & 1.844 & 4,6 & 4.170 & 4,3 \\
\hline $\begin{array}{l}\text { Esquema de tratamento } \\
\text { materno }\end{array}$ & & & & 0,0 \\
\hline Adequado & 1.493 & 3,7 & 3.797 & 4,0 \\
\hline Inadequado & 20.166 & 50,4 & 55.365 & 57,6 \\
\hline Não Realizado & 13.828 & 34,6 & 25.813 & 26,9 \\
\hline Ignorado & 4.521 & 11,3 & 11.117 & 11,6 \\
\hline Parceiro tratado & & & & 0,0 \\
\hline Sim & 4.386 & 11,0 & 13.757 & 14,3 \\
\hline Não & 26.351 & 65,9 & 59.852 & 62,3 \\
\hline Ignorado & 9.271 & 23,2 & 22.483 & 23,4 \\
\hline Fonte: DIAHV/MS/svs, 2019. & & & & \\
\hline & & & & \\
\hline
\end{tabular}

de definição de casos de sífilis adquirida, sífilis em gestante e sífilis congênita, conforme Nota Informativa n 2 SEI/ 2017 DIAHV/ SVS/ MS. A referida norma foi instituída justamente para fins de adequação da sensibilidade na captação de casos de sífilis congênita e reduzir a subnotificação de casos de sífilis em gestantes ${ }^{1,4}$.

No que concerne à sífilis congênita, os resultados deste estudo indicaram que a eliminação da sífilis congênita não foi alcançada, conforme a meta estipulada, em 2010, pela OPAS (Organização Pan-Americana de Saúde), para que os municípios brasileiros reduzissem a taxa de sífilis congênita ao valor menor ou igual a 0,5 casos por 1.000 nascidos vivos, no ano de $2015^{13}$. Ao contrário, em 2017, foram notificados 24.668 casos de sífilis congênita no país, o que significou um aumento de $16,4 \%$ de notificaçôes, em relação ao ano anterior, com uma taxa (nacional) de incidência de sífilis congênita de 8,2 casos por 1000 nascidos vivos no Brasil, e taxas ainda superiores nas regiões Sudeste $(8,9)$ e Sul $(8,8)$.

O aumento no número de casos notificados e da taxa de incidência de sífilis congênita ressalta a necessidade de cuidados pré-natais continuados e da prática diagnóstica de sífilis para todas as mulheres grávidas $^{12}$. No Brasil, o conjunto de estratégias do Ministério da Saúde que visa ampliação do acesso e qualidade do pré-natal na Atenção Básica se apoia na oferta dos testes ráe suas parcerias sexuais, no âmbito da Rede Cegonha, que foi implementada em $2011^{14}$.

Entretanto, estudos demonstraram que a utilização e cobertura de teste rápido de sífilis na Atenção Básica ainda não são satisfatórias. Um estudo realizado em Santa Catarina identificou que dos 123 enferpidos de HIV e de sífilis para as gestantes meiros responsáveis pelas Estratégias Saúde da Família (ESF) do município, embora capacitados, referiram dificuldades de infraestrutura e de recursos humanos para a implantação do serviço de oferta de testes rápidos para sífilis, o que restringiu o diagnóstico de gestantes naquela localidade 5 .

Resultados semelhantes foram observados no Ceará, para a implantação de testes rápidos de sífilis e HIV na rotina do pré-natal, haja vista que a maioria das unidades primárias de saúde contava com profissionais capacitados, porém apresentavam alguma inadequação quanto ao espaço físico e, em muitas, havia indisponibilidade de kits de teste rápido ou até possuíam kits com validade vencida ${ }^{15}$.

Fatores associados à ocorrência da sífilis materna e congênita são objetos de vários estudos e investigações. Algumas características maternas, nível socioeconômico menos favorável e dificuldades de acesso à saúde de qualidade frequentemente são observados nos registros de casos notificados desse agravo ${ }^{12}$.

Os resultados apontaram uma fragilidade de efetividade na assistência pré-natal e no sistema de vigilância epidemiológica do agravo, o que remete a questionamentos, de qual seria a qualidade de assistência prestada a esta população em sua condição de vulnerabilidade ${ }^{11}$.

A idade materna, é fator de decisão sobre o planejamento da gravidez e a qualidade que esta gestação irá vivenciar, depende do contexto social em que a mulher está inserida, já que a gravidez precoce e não planejada, além de condições de vida precárias e em áreas de risco dificultaram a captação e início precoce do pré-natal, além disso, a baixa escolaridade foi um agravante para entendimento da importância dos cuidados à saúde materno-infantil ${ }^{16}$.

Os resultados deste estudo identificaram uma preocupante ineficiência quanto ao tratamento, materno e de seu parceiro, em todo o país. Convém ressaltar que a sífilis congênita e suas consequências podem ser evitadas mediante tratamento adequado da gestante infectada, com a administração de penicilina $G$ benzatina, o único antimicrobiano capaz de atravessar a barreira pla- 
centária e chegar até o feto ${ }^{13}$.

Deve-se ficar atento ainda aos parceiros sexuais, os quais também devem aderir ao tratamento, concomitantemente, para fins de interrupção da cadeia de transmissão. Entretanto, este persiste como um dos maiores empecilhos para o fim do agravo e cura efetiva da gestante ${ }^{13}$.

Neste quesito, há que ressalvar-se que nem sempre a gestante é casada ou desfruta de relacionamento estável e a sífilis pode ser consequência de relacionamentos extraconjugais 17,18. Além disso, devem ser consideradas questões comportamentais e culturais que dificultam a promoção da saúde integral do homem, quanto à procura dos serviços de saúde ${ }^{9,17}$.

\section{CONCLUSÃO}

Apesar dos esforços governamentais realizados para eliminação da sífilis congênita, considerada um marcador da qualidade de assistência à saúde materno-fetal, os resultados deste estudo indicaram que o país se encontra muito distante das metas da Organização Mundial de Saúde, haja vista o progressivo aumento das taxas de incidência em diferentes regiões e sob condições desiguais de acesso à saúde no Estado bra- sileiro.

Convém ressaltar algumas limitações do presente estudo, dada às dificuldades de utilização de dados secundários, possíveis subnotificações de casos, informações consideradas como "ignoradas", falta de acesso aos prontuários e de diferentes fontes de pesquisa. Entretanto, espera-se que os resultados aqui apresentados possam contribuir para uma reflexão em prol da qualidade da atenção à gestante e seu cônjuge ou parceiro sexual, durante o pré-natal, com ênfase nos pontos frágeis já detectados, de modo a subsidiar novas estratégias ou intervenções direcionadas para o controle de casos.

\section{REFERÊNCIAS}

1. Brasil. Ministério da Saúde. Nota Informativa no 2-SEl/2017-DIAHV/SVS/MS [Internet]. Brasília: Ministério da Saúde. 2017 [acesso em 29 apr 2020]. Disponível em: http://portalsinan.saude.gov.br/images/documentos/Agravos/Sifilis-Ges/Nota_Informativa_Sifilis.pdf

2. Brasil. Ministério da Saúde. Protocolo Clínico e Diretrizes Terapêuticas para Prevenção da Transmissão Vertical de HIV, Sífilis e Hepatites Virais. Brasília: Ministério da Saúde. 2019b.

3. WHO. Shortages of benzathine penicillin. How big is the problem? And why it matters [Internet]. Sexual and reproductive health. 2017 [acesso em 29 apr 2020]. Disponivel em: https://www.who.int/reproductivehealth/shortages-benzathine-penicillin/en/

4. Brasil. Boletim epidemiológico [Internet]. v. 49. Brasilia: Ministério da Saúde; 2018 [acesso em 29 apr 2020]. Disponível em: http:// www.aids.gov.br/pt-br/pub/2018/boletim-epidemiologico-de-sifilis-2018/

5. Nascimento DDSF, Silva RC, Tártari DDO, Cardoso ÉK. Relato da dificuldade na implementação de teste rápido para detecção de sífilis em gestantes na Atenção Básica do SUS em um município do Sul do Brasil. Rev Bras Med Família e Comunidade. 2018;13(40):1-8.

6. Nunes JPG, Barbosa VV. A historical series of the incidence of Syphilis in pregnant in Pará between 2013 to 2018. Para Res Med J. 2020;4:e32.

7. Brasil. Conselho Nacional de Saúde. Resolução no 466, de 12 de dezembro de 2012. [Internet]. Brasília: Ministério da Saúde. 2012 [acesso em 29 apr 2020]. Disponivel em: https://conselho.saude.gov. br/resolucoes/2012/ Reso466.pdf

8. Brasil. Conselho Nacional de Saúde. Resolução No 510, de 07 de abril de 2016. Brasília: Ministério da Saúde. 2016 [acesso em 29 apr 2020]. Disponivel em: http://conselho.saude.gov.br/resolucoes/2016/Reso510.pdf.

9. Brasil. Indicadores e Dados Básicos da Sífilis nos Municípios Brasileiros [Internet]. 2018 [acesso em 29 apr 2020]. Disponível em: http://indicadoressifilis.aids.gov.br/
10. Saraceni V, Pereira GFM, Da Silveira MF, Araujo MAL, Miranda AE. Vigilância epidemiológica da transmissão vertical da sífilis: Dados de seis unidades federativas no Brasil. Rev Panam Salud Publica/Pan Am J Public Heal . 2017;41(1):1-8.

11. Lafetá KRG, Martelli Júnior H, Silveira MF, Paranaíba LMR. Maternal and congenital syphilis, underreported and difficult to control. Rev Bras Epidemiol. 2016;19(1):63-74.

12. Padovani C, Oliveira RR, Pelloso SM. Syphilis in during pregnancy: Association of maternal and perinatal characteristics in a region of southern Brazil. Rev Lat Am Enfermagem. 2018;26(e3019):1-10.

13. Brasil. Caderno de boas práticas: o uso da penicilina na Atenção Básica para a prevenção da sífilis congênita no Brasil. v. 1. Brasília: Ministério da Saúde; 2015.

14. Brasil. Portaria no 1.459 , de 24 de junho de 2011. [Internet]. Diário Oficial da União; 2011 [acesso em 29 ago 2020]. Disponivel em: https:/bvsms.saude.gov.br/bvs/saudelegis/gm/2011/ prt1459_24_06_2011.html

15. Lopes ACMU, Araújo MAL, Vasconcelo LDPG, Uchoa FSV, Rocha HP, Santos JR. Implantação dos testes rápidos para sífilis e HIV na rotina do pré-natal em Fortaleza - Ceará. Rev Bras Enferm. 2016; 69(1):62-6.

16. Nonato SM, Melo APS, Guimarães MDC. Sífilis na gestação e fatores associados à sífilis congênita em Belo Horizonte-MG, 20102013. Epidemiol e Serviços Saúde. 2015; 24(4):681-94.

17. Fontes MB, Crivelaro RC, Scartezini AM, Lima DD, Garcia ADA, Fujioka RT. Determinant factors of knowledge, attitudes and practices regarding STD/AIDS and viral hepatitis among youths aged 18 to 29 years in Brazil. Cienc e Saude Coletiva. 2017; 22(4):1343-52.

18. Bernardo MRS, Pinto EO, Costa ET, Armada e Silva HCD, Bernardo da Silva R, Santos MF, Chícharo SCR. Conhecimento sobre a sífilis e o acompanhamento de gestantes em uma clínica da família da Zona Oeste do Rio de Janeiro. SaudColetiv (Barueri). 2020; 10(52):212437. 